\title{
Use of Bedside Ultrasound in the Emergency Department as Preliminary Diagnostic Workup for a Patient with Pericardial Effusion
}

\author{
Javad Najjar Mojarrab ${ }^{1 *}$, Nasvin Imamudeen ${ }^{1}$, Somto Nwaedozie ${ }^{1}$, Shivy Sharma ${ }^{1}$ and Abuzaid Medani ${ }^{2}$ \\ ${ }^{1}$ Department of Internal Medicine, Marshfield Clinic Health System, USA
}

${ }^{2}$ Department of Hospital Medicine, Marshfield Clinic Health System, USA

Submission: February 19, 2021; Published: March 09, 2021

*Corresponding author: Javad Najjar Mojarrab, MD, MBA, Department of Internal Medicine, Marshfield Clinic Health System - Marshfield, 1,000 North Oak Avenue 3K2 Marshfield, WI 54449, Wisconsin, USA

\section{Abstract}

Pericardial effusion occurs when there is approximately 150-200 cubic centimeters (cc) of fluid in the pericardium. It can be a manifestation of disease processes such as pericarditis, cancer, trauma, or aortic dissection. Management of pericardial effusion is dependent on whether there is hemodynamic instability. Here, we present a case of a 63-year-old man who presented with a progressively worsening bilateral lower extremity edema and oliguria for two weeks and was found to have a very large circumferential pericardial effusion via bedside ultrasonography. This case highlights the importance of bedside ultrasonography in detecting and decreasing time to intervention in pericardial effusions with hemodynamic instability.

Keywords: Pericardial effusion; Cardiac tamponade; Bedside ultrasound; Malignant pericardial effusion; NSCLC

Abbreviation: NSCLC: Non-Small Cell Lung Cancer; CC: Cubic Centimeters; MRI: Magnetic Resonance Imaging; POCUS: Point-of-Care Ultrasound

\section{Introduction}

Lung cancer is the most common cause of cancer deaths worldwide. The primary subtypes of lung cancer are non-small cell lung cancer (NSCLC) and small cell lung cancer with the former comprising $80 \%$ of lung cancer cases [1]. Non-small cell lung cancer further subdivides into adenocarcinoma, squamous cell carcinoma, and large cell carcinoma [1]. The most common presenting symptoms of lung cancer include weight loss, cough, hemoptysis, dyspnea, weakness, and chest pain [2]. NSCLC has been known to cause malignant pericardial effusion in approximately $7.4 \%-7.8 \%$ of cases which is associated with a high recurrence rate and poor prognosis [3-5]. Pericardial effusion is defined as the presence of more than 150 to $200 \mathrm{cc}$ of fluid in the pericardium [6]. Regardless of its etiology, pericardial effusion can lead to the development of life-threatening cardiac tamponade that compresses the heart and prevents filling of the heart during diastole. Clinical signs associated with cardiac tamponade include tachycardia, hypotension, muffled heart sounds, and elevation of jugular venous pressure. Besides clinical acumen, diagnostic testing such as echocardiography, computed tomography, or magnetic resonance imaging (MRI) could help in diagnosing a pericardial effusion [7].

In a recent case report of a patient with malignant pericardial effusion, Rosario and colleagues report that use of a bedside ultrasound may diagnose pericardial effusion much quicker than other diagnostic imaging technologies [8]. We present a case of a 63-year-old patient who exhibited worsening bilateral lower extremities and oliguria of two weeks duration. A bedside ultrasound in the emergency department revealed a large pericardial effusion with signs of hemodynamic compromise that was later diagnosed as NSCLC through pathology workup. This case highlights the importance of bedside ultrasound in quickly diagnosing pericardial effusion, and we recommend further study to evaluate the diagnostic performance of this rapid imaging modality for patients with suspected pericardial effusion. 


\section{Case Presentation}

A 63-year-old man with a history of untreated hypertension who had not seen a physician for more than a decade and chronic smoking history of $40+$ pack years presented to the emergency department with a two-week history of worsening bilateral lower extremity edema and increased swelling of his lower extremities with pitting for two days. The patient also noticed that his urine output was less than usual for the past month. He did not have any chest pain, shortness of breath, exertional dyspnea, orthopnea, paroxysmal nocturnal dyspnea, or reported history of recent illness. At baseline physical examination, the patient was active and able to perform activities of daily living.

At the time of admission, he was found to have an elevated blood pressure of $260 \mathrm{mmHg} / 120 \mathrm{mmHg}$. Initial labs revealed elevated levels of alkaline phosphatase (143 units (U)/L \{normal: 43-115 U/L\}), aspartate aminotransferase (54 U/L \{normal: 13$39\})$, total bilirubin $(2.3 \mathrm{mg} / \mathrm{dL}$ normal: $0.1-1.0 \mathrm{mg} / \mathrm{dL}\})$, and brain natriuretic peptide $(139 \mathrm{pg} / \mathrm{mL}$ \{normal: $0-80 \mathrm{pg} / \mathrm{mL}\})$. The patient's sodium and potassium levels were slightly below normal (131 mmol/L \{normal: $133-144 \mathrm{mmol} / \mathrm{L}\}$ and $3.2 \mathrm{mmol} / \mathrm{L}$ \{normal: 3.4-5.1mmol/L\}, respectively). An initial electrocardiogram revealed sinus tachycardia, and a chest X-ray suggested severe cardiomegaly with asymmetric opacity in the right lung apex (Figure 1). The patient was admitted for a hypertensive emergency secondary to high blood pressure and elevated liver enzymes. No other signs of end-organ damage were present.

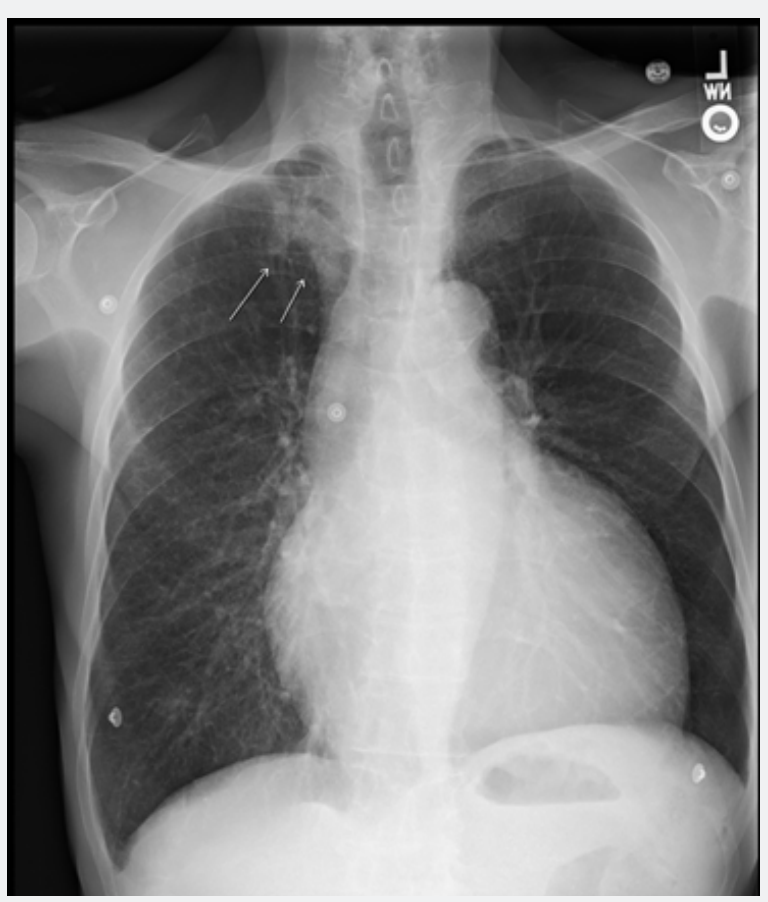

Figure 1: Severe cardiomegaly and asymmetric opacity in the medial right lung apex.

Later on, in the day of admission, a preliminary bedside echocardiogram was performed which suggested a large pericardial effusion. Further echocardiographic imaging was performed which revealed a very large circumferential pericardial effusion with signs of hemodynamic compromise (Figure 2). Our patient underwent urgent pericardiocentesis which drained $1.8 \mathrm{~L}$ of hemorrhagic fluid. Cytology of pericardial effusion revealed malignant cells derived from poorly differentiated metastatic adenocarcinoma which was strongly positive for epithelial specific antigen/epithelial cell adhesion molecule (detected using MOC31 antibody), and negative for thyroid transcription factor- 1 and Napsin A with $0 \%$ staining of cells for programmed death ligand 1.
A computed tomography of the chest with contrast (Figure 3) showed a large pericardial effusion and abnormal irregular nodule in the right lung apex with para-tracheal, hilar, and abnormal appearing right supraclavicular lymph node indicative of lung cancer. Further workup with positron emission tomography and computed tomography of the chest showed findings consistent with lung malignancy. MRI of the brain did not reveal any metastasis though detected extensive microvascular changes with numerous lacunar infarcts. Two days after the initial pericardiocentesis, repeat echocardiogram revealed a reaccumulation of the pericardial effusion circumferentially without evidence of increased intra-pericardial pressures. 


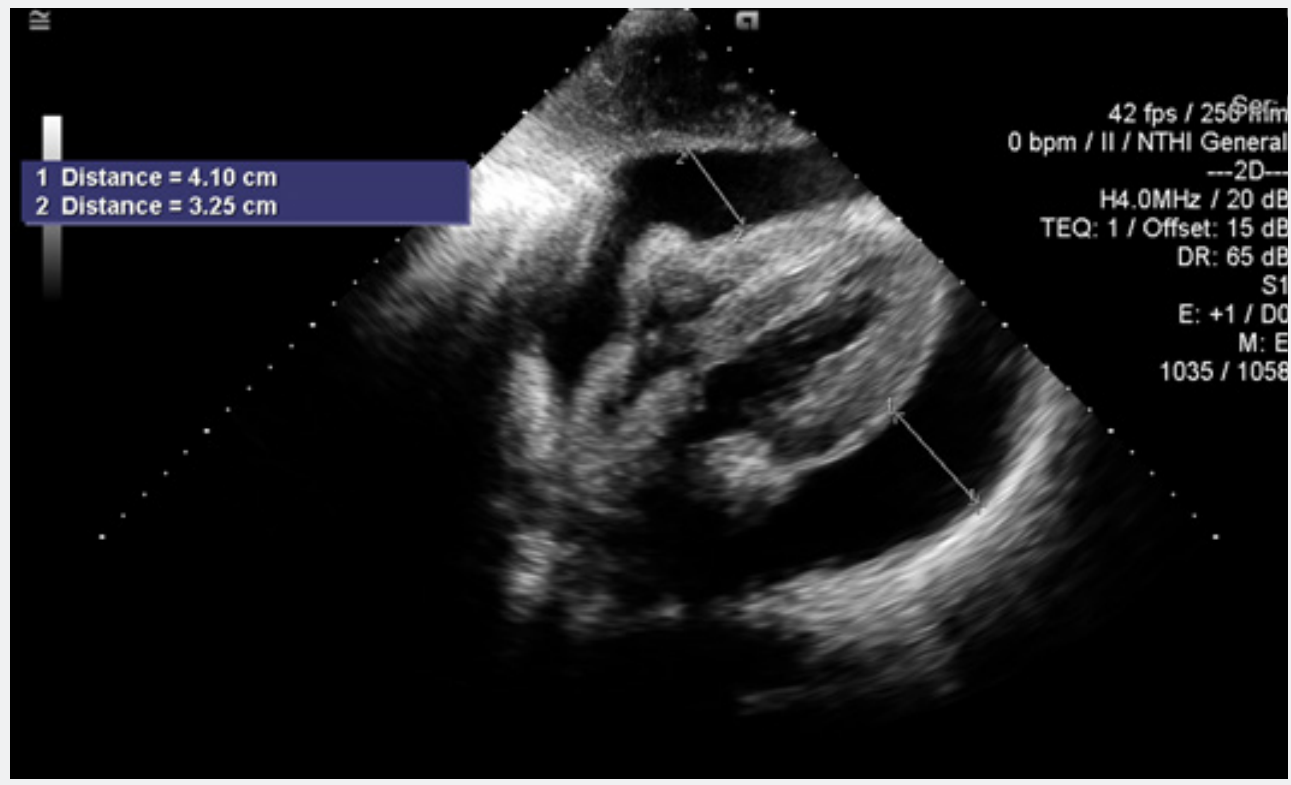

Figure 2: A very large circumferential pericardial effusion visualized via echocardiogram.

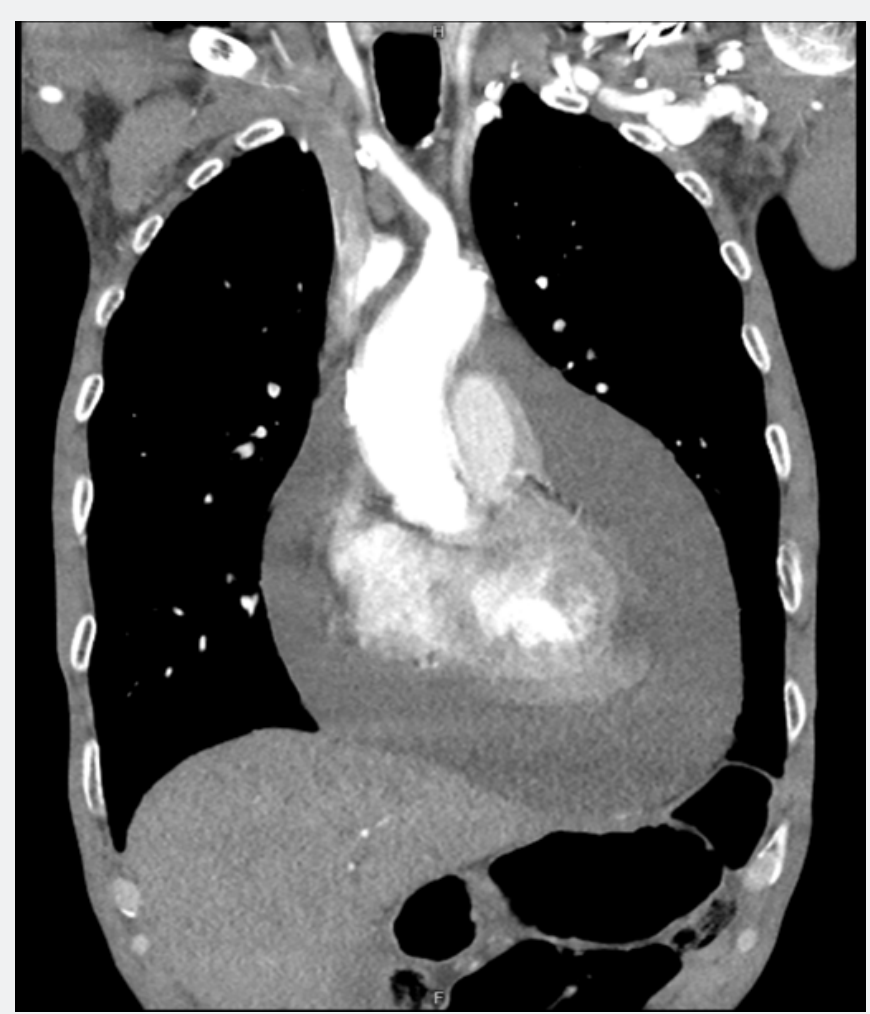

Figure 3: Computed tomography of the chest showing a very large pericardial effusion.

Consecutive laboratory testing throughout the patient's stay revealed hyponatremia as low as $125 \mathrm{mmol} / \mathrm{L}$, increased urine osmolality of $429 \mathrm{mOs} / \mathrm{kg}$, and decreased serum sodium at
$129 \mathrm{mmol} / \mathrm{L}$ suggesting syndrome of inappropriate antidiuretic hormone secretion secondary to lung malignancy. The patient was managed with fluid restriction of $1,500 \mathrm{cc} /$ day and was started on a 
combination regimen of chemotherapy and immunotherapy with carboplatin, pemetrexed, and pembrolizumab. The patient was discharged home with a scheduled follow-up with a cardiothoracic surgeon for an outpatient pericardial window procedure. Unfortunately, the patient passed away due to complications from his NSCLC before this procedure was completed.

\section{Discussion}

Approximately 7.4-7.8 \% of patients with NSCLC have associated malignant pericardial effusion which has been shown to have a poorer prognosis with a median survival time of six months [3-5]. Pericardial effusion is confirmed to be an independent prognostic factor of lung cancer death [3]. The primary treatment for pericardial tamponade secondary to NSCLC is pericardiocentesis and/or pericardial window; however, Lestuzzi et al. [9] reported that a combination regimen of intrapericardial chemotherapy with pericardiocentesis has resulted in a better prognosis and improved response [9]. However, this treatment combination was not possible in our patient since he had a very progressive disease and passed away after the second day of cycle one of his NSCLC treatment regimen.

Expediting time to diagnosis and initiation of therapy in pericardial effusion with tamponade physiology is very crucial not just in decreasing morbidity and mortality but also for improving overall patient outcomes [10,11]. Point-of-care ultrasound (POCUS) plays a vital role in the initial evaluation and prompt management of patients presenting with cardiovascular symptoms because it narrows the differential diagnosis and guides the initiation of goal-directed therapy and subspecialty consultations. It is a reliable tool of high diagnostic utility especially in the setting of cardiac tamponade or pericardial effusion where it has been demonstrated to have a sensitivity of $96 \%$ and a specificity of $98 \%$ [12]. In a retrospective cohort study of the utility of POCUS in the emergency setting, Alpert et al. observed that compared to other diagnostic modalities, POCUS decreased time to pericardiocentesis and length of hospitalization, and as a result, its use should be considered in the initial work-up of patients presenting with cardiovascular symptoms [13].

The differential diagnoses for a sub-acute bilateral pitting leg edema, which was the initial presentation of our patient, includes heart failure, liver disease, renal pathologies, and bilateral venous thrombosis. As our patient had not had a medical encounter for more than 15 years, the differential diagnoses for his presentation were broader. In the context of his untreated hypertension, the presence of pulmonary vascular congestion on chest X-ray and cardiomegaly, the clinical suspicion of diastolic heart failure was high. Since the patient presented with hypertensive urgency, concern for cardiac tamponade or clinically significant pericardial effusion, which typically presents with hypotension, was not among the top differentials. The prompt use of bedside POCUS was crucial in narrowing the differential diagnosis as well as initiating lifesaving pericardiocentesis before further etiological work-up was completed.

\section{Conclusion}

Prompt identification of pericardial effusion in the context of hemodynamic instability is necessary for the initiation of timely, adequate treatment. In this patient, prompt use of POCUS decreased time to intervention and avoided the initiation of potentially detrimental therapeutics like diuretics and use of antihypertensive medications. Use of POCUS should be considered for patients with symptoms suggestive of pericardial effusion.

\section{Acknowledgement}

The authors acknowledge Emily Andreae, PhD, from the Marshfield Clinic Research Institute for manuscript editing assistance.

\section{References}

1. Zalcman G, Bergot E, Lechapt E (2010) Update on nonsmall cell lung cancer. European Respiratory Review 19: 173-185.

2. Chute CG, Greenberg ER, Baron J, Korson R, Baker J, et al. (1985) Presenting conditions of 1539 population-based lung cancer patients by cell type and stage in New Hampshire and Vermont. Cancer 56(8): 2107-2111.

3. Hu ZG, Hu K, Li WX, Zeng FJ (2019) Prognostic factors and nomogram for cancer-specific death in non small cell lung cancer with malignant pericardial effusion. PLoS ONE 14(5): e0217007.

4. Ou SHI, Zell JA (2008) Validation study of the proposed IASLC staging revisions of the T4 and $\mathrm{M}$ non-small cell lung cancer descriptors using data from 23,583 patients in the California Cancer Registry. J Thorac Oncol 3(3): 216-227.

5. Kocher F, Fiegl M, Mian M, Hilbe W (2015) Cardiovascular comorbidities and events in NSCLC: Often underestimated but worth considering. Clin Lung Cancer 16(4): 305-312.

6. Engel PJ, Hon H, Fowler NO, Plummer S (1982) Echocardiographic study of right ventricular wall motion in cardiac tamponade. Am J Cardiol 50(5): 1018-1021.

7. Restrepo CS, Lemos DF, Lemos JA, Velasquez E, Diethelm L, et al. (2007) Imaging findings in cardiac tamponade with emphasis on CT. Radiographics 27(6): 1595-1610.

8. Rosario J, Mangal R, Houck J, Slome MC, Ganti L (2020) Pericardial effusion with tamponade: Bedside ultrasonography saves another life. Int J Emerg Med 13(1): 3.

9. Lestuzzi C, Bearz A, Lafaras C, Gralec R, Cervesato E, et al. (2011) Neoplastic pericardial disease in lung cancer: Impact on outcomes of different treatment strategies. A multicenter study. Lung Cancer 72(3): 340-347.

10. Sebat F, Musthafa AA, Johnson D, Kramer AA, Shoffner D, et al. (2007) Effect of a rapid response system for patients in shock on time to treatment and mortality during 5 years. Crit Care Med. 35(11): 25682575.

11. Jones AE, Tayal VS, Sullivan DM, Kline JA (2004) Randomized, controlled trial of immediate versus delayed goal-directed ultrasound to identify the cause of nontraumatic hypotension in emergency department patients. Crit Care Med 32(8): 1703-1708. 
12. Mandavia DP, Hoffner RJ, Mahaney K, Henderson SO (2001) Bedside echocardiography by emergency physicians. Ann Emerg Med 38(4) 377-382.
13. Alpert EA, Amit U, Guranda L, Mahagna R, Grossman SA, et al. (2017) Emergency department point-of-care ultrasonography improves time to pericardiocentesis for clinically significant effusions. Clin Exp Emerg Med 4(3): 128-132.
- Quality Editorial service

- Swift Peer Review

- Reprints availability

- E-prints Service

- Manuscript Podcast for convenient understanding

- Global attainment for your research

- Manuscript accessibility in different formats ( Pdf, E-pub, Full Text, Audio)

- Unceasing customer service

Track the below URL for one-step submission https://juniperpublishers.com/online-submission.php 